\title{
Flying Blind: Early Outcomes Following Implementation of Formal Critical Care Services in a Low-Resource Setting
}

\author{
Elizabeth M Stoeckl, BS ${ }^{1}$, Kristin L Long, MD, $M P H^{1^{*}}$ and Emnet Tesfaye, $M D^{2}$ \\ ${ }^{1}$ Division of Endocrine Surgery, University of Wisconsin-Madison, USA \\ ${ }^{2}$ Hawassa University Hospital, Ethiopia
}

*Corresponding author: Kristin L Long, MD, MPH, Division of Endocrine Surgery, Department of Surgery, University of Wisconsin School of Medicine and Public Health, 600 Highland Avenue Madison, WI 53792-7375, USA, Tel: 608-263-1387, Fax: 608-263-7652

\begin{abstract}
Global health endeavors in low- and middle-income countries (LMIC) have traditionally emphasized humanitarian relief and primary care. Increasingly, specialty services, such as critical care medicine, are recognized as key components of sustainable, effective health care systems, even in settings with constrained resources. We illustrate the successful implementation of critical care services at Hawassa University teaching hospital in Ethiopia. Although not without challenges, the introduction of specialized medical care can be both feasible and impactful in low-resource communities. In the two years since a critical care-trained physician joined the hospital staff, the mortality rate of critically ill patients improved by $11 \%$. These early outcomes argue in favor of continued attempts to initiate and research the implementation of critical care services in novel settings, with particular attention to partnerships between institutions, organizations and local physicians.
\end{abstract}

\section{Keywords}

Critical care, Global health, Ethiopia, Low- and middle-income countries

\section{Introduction}

Since its inception during the poliomyelitis epidemic in the early $20^{\text {th }}$ century, critical care medicine has focused on continuous, multidisciplinary care for patients with organ insufficiency and life-threatening illness [1]. The critical care model has gained worldwide popularity. However, there remains a large gap in necessary care in low- and middle-income countries (LMIC), where the global burden of critical illness disproportionately falls [2]. Most deaths from treatable causes occur in LMIC, including $90 \%$ of global trauma-related mortality [3] $94 \%$ percent of global maternal mortality [4], and sequelae of sepsis and other potentially reversible acute conditions. Compared to high-income countries (HIC), critically ill patients in LMIC are frequently younger and have fewer comorbidities [5]. Thus, strengthening critical care services offers the possibility for significant population health and economic gains.

Although the unmet need is evident, little data exist to assess the introduction and outcomes of critical care programs in resource-limited settings [6-9]. More than $50 \%$ of LMICs lack any published data on their intensive care unit (ICU) capacity [10], and the World Health Organization does not track capacity to care for critically ill individuals or global ICU bed availability [2]. In order to revisit the challenges and opportunities in providing critical care in low-resource areas, we illustrate the clinical outcomes at one hospital in Hawassa, Ethiopia before and after the addition of an intensivist physician to the hospital staff.

\section{Methods and Results}

The Hawassa University teaching hospital represents the major referral center for Southern Ethiopia, with a catchment area of approximately 20 million individuals. Within the hospital, a seven-bed critical care unit was opened approximately three years ago (October 2016). In the initial two years and ten months that the ICU facility was open, over one thousand patients were admitted to the unit, and 471 patients died. In

Citation: Stoeckl EM, Long KL, Tesfaye E (2020) Flying Blind: Early Outcomes Following Implementation of Formal Critical Care Services in a Low-Resource Setting. Int J Crit Care Emerg Med 6:106. doi. org/10.23937/2474-3674/1510106

Accepted: May 12, 2020: Published: May 14, 2020

Copyright: (C) 2020 Stoeckl EM, et al. This is an open-access article distributed under the terms of the Creative Commons Attribution License, which permits unrestricted use, distribution, and reproduction in any medium, provided the original author and source are credited. 
Table 1: Reduction in total mortality after arrival of critical care specialist physician in Hawassa University ICU.

\begin{tabular}{|l|l|l|}
\hline & Pre-MD & Post-MD \\
\hline Deaths & 471 & 144 \\
\hline Total Admissions & 1081 & 430 \\
\hline Total Mortality & $44 \%$ & $33 \%$ \\
\hline
\end{tabular}

Fall 2017, a critical care-trained physician, Dr. Tesfaye (ET), was hired to manage the ICU patients in attempt to improve quality of care and overall survival. Since her arrival, the total number of patients admitted to the ICU has been 430, with 144 patients dying. Of the deceased patients under Dr. Tesfaye's care, 116 remained in the ICU for less than one week, 18 for 8-14 days, and five for greater than two weeks. With very limited records available, comprehensive chart analysis is challenging. At present, the arrival of a critical care specialist demonstrates at least an $11 \%$ improvement in overall mortality for the critically ill patients treated at Hawassa University (Table 1).

Although these data are rudimentary, they speak powerful evidence to the benefits of supporting critical care services within hospitals in low-resource settings. There are many challenges, however, to providing quality care in such resource constrained facilities. A tremendous variety of critically ill patients present to Hawassa frequently, and the ability to transfer some patients to higher levels of care does exist. In addition, as responsible stewards of the limited ICU resources, the Hawassa critical care team often faces difficult patient scenarios. For example, one of the valuable seven ICU beds available in the facility has been occupied for over one year with a stable, alert, but ventilator-dependent young patient who cannot be safely transferred to a regular floor bed or transferred home. The resources and physical space alone are often limiting factors.

\section{Discussion}

While representative of just one institution, Dr. Tesfaye's perspectives from Hawassa add to the growing body of evidence supporting specialty services as a key component of sustainable health systems strengthening [11]. Traditional approaches to public health have focused on primary care development, preventative health measures, and disease-specific elimination. However, building sustainable health systems concurrently-including investing in human resources and facilities-augments the effectiveness of public health initiatives [12]. While trauma, HIV, infectious diseases, and obstetric complications are theoretically preventable with robust prevention and primary care, they continue cause significant mortality. A lack of post-prevention infrastructure, including critical care, represents an opportunity lost.

Critical care has popularly been considered too costly and complex to bring to scale in low-resource settin- gs. However, the infrastructural components essential to critical care-microbiological studies, imaging, invasive monitoring, water, electricity, and consistent oxygen supply-are arguably integral to providing quality care in any specialty, and they should be included in hospital development plans as feasible. Four elements are frequently cited as essential components of both critical care capacity and disaster-preparedness strategy more broadly: Staff, stuff, space, and systems [13]. Hawassa exemplifies how investments in all four of these facets can have a profound impact. Even after appropriate space and equipment were designated for an ICU, Dr. Tesfaye demonstrates that an intensivist-driven team can produce marked improvements in clinical outcomes, underscoring the importance of support for in country staff.

From a systems perspective, multiple quality improvement endeavors have also been instituted since the introduction of trained critical care staff. Hawassa University serves as the inaugural facility for an initiative led by the American College of Surgeons Operation Giving Back team, in which a consortium of universities from HICs partner together to provide continual staffing and educational efforts. The partnership with several visiting critical care surgeons has led to the introduction of hand hygiene protocols (with hand-sanitizing equipment at each bed), advanced cardiac life support resuscitation training for the ICU nurses, and teaching sessions for all ICU staff on various topics.

Given the incredible volume of patients seen at this facility, running a critical care unit will rapidly become unsustainable for just one in-country physician. However, the progress already made in Hawassa demonstrates that the introduction of critical care services in a low-resource setting is both feasible and effective. The establishment of additional in-country critical care training fellowships, ongoing multi-institutional partnerships, and hospital-level support of additional trained staff will help further address this issue; however, it is imperative that it remain a priority among topics in clinical research and development.

\section{Conflict of Interest}

The authors report no proprietary or commercial interest in any product mentioned or concept discussed in this article. There are no disclosures to report.

\section{Author Contributions}

EMS, KLL, and ET designed the study. ET collected the data. EMS, KLL, ET analyzed the data. EMS, KLL, ET wrote and revised the manuscript.

\section{References}

1. Marshall JC, Bosco L, Adhikari NK, Connolly B, Diaz JV, et al. (2017) What is an intensive care unit? A report of the task force of the World Federation of Societies of Intensive and Critical Care Medicine. J Crit Care 37: 270-276. 
2. Fowler RA, Adhikari NK, Bhagwanjee S (2008) Clinical review: Critical care in the global context-disparities in burden of illness, access, and economics. Crit Care 12: 225.

3. Mock C, Kobusingye O, Joshipura M, Nguyen S, Arreola-Risa C (2005) Strengthening trauma and critical care globally. Curr Opin Crit Care 11: 568-575.

4. WHO (2019) Trends in maternal mortality: 2000 to 2017. Geneva: World Health Organization.

5. Riviello ED, Letchford S, Achieng L, Newton MW (2011) Critical care in resource-poor settings: Lessons learned and future directions. Crit Care Med 39: 860-877.

6. Baker T (2009) Critical care in low-income countries. Trop Med Int Health 14: 143-148.

7. Vukoja M, Riviello E, Gavrilovic S, Adhikari NK, Kashyap $R$, et al. (2014) A survey on critical care resources and practices in low- and middle-income countries. Glob Heart 9: 337-342.

8. Fowler RA, Adhikari NK, Sacles DC, Lee WL, Rubenfield GD (2008) Update in critical care 2007. Am J Respir Crit Care Med 177: 808-819.
9. Smith ZA, Ayele Y, McDonald P (2013) Outcomes in critical care delivery at Jimma University Specialised Hospital, Ethiopia. Anaesth Intensive Care 41: 363-368.

10. Murthy S, Leligdowicz A, Adhikari NK (2015) Intensive care unit capacity in low-income countries: A systematic review. PLoS One 10: e0116949.

11. Meara JG, Leather AJ, Hagander L, Alkire BC, Alonso N, et al. (2015) Global surgery 2030: Evidence and solutions for achieving health, welfare, and economic development. Lancet 386: 569-624.

12. Palen J, El-Sadr W, Phoya A, Imtiaz R, Einterz R, et al. (2012) PEPFAR, health system strengthening, and promoting sustainability and country ownership. J Acquir Immune Defic Syndr 60: S113-S119.

13. Khajehaminian MR, Ardalan A, Keshtkar A, Hosseini Boroujeni SM, Nejati A, et al. (2018) A systematic literature review of criteria and models for casualty distribution in trauma related mass casualty incidents. Injury 49: 1959-1968. 